\title{
Multidisciplinary heart failure clinics: Are they effective in Canada?
}

\author{
Michael W. Rich
}

B See related article page 40

A $\mathrm{s}$ in most Western countries, the burden of heart failure in Canada is increasing, primarily because of the aging of the population, but also in part because of improved survival among patients with hypertension and coronary artery disease. In Montreal, the number of hospital admissions because of heart failure among people 65 years and older increased from 2299 in 1990/91 to 3339 in $1997 / 98$, reflecting a $35 \%$ increase in the rate of heart failure admissions per 10000 population. ${ }^{1}$ Nationwide, heart failure affects more than 400000 Canadians, and over 50000 new cases are diagnosed each year. ${ }^{2}$ Moreover, despite major advances in treatment over the past 25 years, there have been only modest improvements in heart failure outcomes on a population-wide basis. For example, in 2000/01, there were 106130 admissions of 85679 patients with congestive heart failure to Canadian hospitals. Of these, $32.7 \%$ were readmissions, and in-hospital mortality was $15.8 \%$. Of patients discharged alive, $19.9 \%$ were readmitted at least once before Mar. 31, 2001. ${ }^{3}$ These statistics, although sobering, mirror trends reported in Europe and the United States.

What accounts for the persistently high rate of hospital admissions among patients with heart failure? Disease severity is an important factor: patients with advanced heart failure have a very limited cardiovascular reserve, and, as a result, modest challenges to hemodynamic stability, such as myocardial ischemia, uncontrolled hypertension or a bout of pneumonia, are sufficient to trigger an acute exacerbation of heart failure. Other factors that often contribute to worsening heart failure include underuse (both underprescription and underdosing) of proven heart failure therapies, such as angiotensin-converting-enzyme inhibitors and $\beta$-blockers; inadequate patient education about the nature of heart failure and the role of self-management; nonadherence to the heart failure medication and dietary regimen; an inadequate social support network; and insufficient follow-up. ${ }^{4,5}$ Importantly, most heart failure readmissions are related primarily to the behavioural and social factors noted above, rather than to acute medical illness. ${ }^{4,5}$

In the past 15 years, there has been increasing recognition of the critical role of patient-centred care in the management of chronic illnesses, including heart failure, diabetes, chronic lung disease and many other conditions. Patient-centred care implies a holistic approach, one that entails much more than simply making the right diagnosis and prescribing the right medications. Rather, patient- centred care mandates that the patient and family, who have a vested interest in the patient's health, be actively engaged in the daily management of the patient's illness. To maximize the likelihood of successful outcomes, close collaboration between the patient and health care team is required; specifically, the patient needs to become empowered through appropriate education about the disease process and treatment, and follow-up needs to be both proactive and more frequent than what occurs with traditional office-based ambulatory care. Furthermore, since most physicians have neither the time nor the expertise to comprehensively address the myriad needs of patients with complex chronic illnesses, a multidisciplinary care model, usually involving physicians and nurses, but often including a pharmacist, social worker, home health specialist, dietician and therapists, has evolved as the preferred strategy for optimizing patient-centred care.

\section{...in 2000/01, there were $106130 \mathrm{admissions}$ of 85679 patients with congestive heart failure to Canadian hospitals. Of these, $32.7 \%$ were readmissions, and in-hospital mortality was $\mathbf{1 5 . 8} \%$. Of patients discharged alive, $19.9 \%$ were readmitted at least once before Mar. 31, 2001.}

The value of multidisciplinary care in the management of patients with heart failure has been assessed in a series of small- to moderate-sized randomized clinical trials, and the results of these studies have recently been reviewed. Two meta-analyses have demonstrated that multidisciplinary heart failure disease management programs, as they are often called, consistently reduce readmission rates by about $25 \%-30 \%$ and have favourable effects on quality of life, cost of care, and survival. ${ }^{6,7}$ To date, however, few studies have been conducted in Canada, where publicly funded health care is provided at no out-of-pocket cost to the user and where access to care is unrestricted; therefore, the applicability of studies conducted in other countries to the care of heart failure patients in Canada remains uncertain.

In this issue of $C M A \mathcal{A}$, Ducharme and colleagues report 
the results of a prospective randomized clinical trial involving 230 patients discharged from hospital with heart failure at the Montreal Heart Institute from 1998 to $2000{ }^{8} \mathrm{~Pa}$ tients received standard post-discharge care, as directed by their primary care physician, or standard care supplemented by a comprehensive and individualized patient education program and frequent follow-up provided by a multidisciplinary team that included cardiologists, nurses, a pharmacist and a dietician. The mean age of the study participants was 69 years, $28 \%$ were women, and there was a high prevalence of coronary artery disease, hypertension and diabetes, as is typical in an older heart failure population. The main findings were that at 6-month follow-up, there was a $45 \%$ reduction in the number of patients admitted to hospital, $32 \%$ reduction in the total number of hospital admissions, and $44 \%$ reduction in the total number of hospital days among patients in the treatment group compared with patients in the control group. In addition, quality of life was significantly improved for patients in the treatment group, and there were fewer deaths, although this difference did not achieve statistical significance.

These findings, which are remarkably consistent with earlier reports, clearly indicate that the benefits of a welldesigned heart failure management program extend to patients receiving care in the publicly funded Canadian health care system. An important limitation of the study, however, is that it was conducted at an academic medical centre with an established heart failure clinic. The generalizability of the findings to other heart failure populations, especially those with fewer medical resources (e.g., no local heart failure clinic) remains unknown. In addition, only 19\% of potentially eligible subjects were actually enrolled in the trial, while a surprisingly high proportion, $65 \%$, refused to participate. Although the reasons for refusal are not provided, it may be inferred that the study population represents a select subgroup of the heart failure population, perhaps a group more amenable to this type of intervention, and it may therefore be injudicious to extrapolate the study findings to the broader heart failure community. As with other similar studies, the present trial also leaves open several key questions, including the optimal intensity and duration of heart failure management programs, which patients derive the greatest benefit (and, conversely, which patients are unlikely to benefit), whether such interventions are cost-effective during long-term follow-up, and how best to translate the results of clinical trials into routine patient care. ${ }^{9}$ Ongoing and future studies should provide further insight into these issues, thereby allowing clinicians to individually tailor care of their heart failure patients to maximize outcomes while minimizing costs. In the meantime, the report by Ducharme and colleagues provides compelling support for the Canadian Cardiovascular Society's recommendations regarding the role of multidisciplinary clinics in the management of patients with congestive heart failure. ${ }^{10}$

Dr. Rich is an Associate Professor of Medicine at Washington University School of Medicine, St. Louis, Mo.

Competing interests: None declared.

\section{References}

1. Feldman DE, Thivierge C, Guérard L, Dery V, Kapetanakis C, Lavoie G, et al. Changing trends in mortality and admissions to hospital for elderly patients with congestive heart failure in Montreal. CMAf 2001;165(8):1033-6.

2. Kostuk WJ. Congestive heart failure: what can we offer our patients? CMAJ 2001;165(8):1053-5.

3. Tsuyuki RT, Shibata MC, Nilsson C, Hervas-Malo M. Contemporary burden of illness of congestive heart failure in Canada. Can 7 Cardiol 2003;19:436-8.

4. Vinson JM, Rich MW, Shah AS, Sperry JC. Early readmission of elderly patients with congestive heart failure. 7 Am Geriatr Soc 1990;38:1290-5.

5. Tsuyuki RT, McKelvie RS, Arnold JM, Avezum A Jr, Barretto AC, Carvalho $\mathrm{AC}$, et al. Acute precipitants of congestive heart failure exacerbations. Arch Intern Med 2001;161(19):2337-42.

6. Phillips CO, Wright SM, Kern DE, Singa RM, Shepperd S, Rubin HR. Comprehensive discharge planning with postdischarge support for older patients with congestive heart failure: a meta-analysis. 7AMA 2004;291:1358-67.

7. McAlister FA, Stewart S, Ferrua S, McMurray JJ. Multidisciplinary strategies for the management of heart failure patients at high risk for admission: a systematic review of randomized trials. $\mathcal{F}$ Am Coll Cardiol 2004;44:810-9.

8. Ducharme A, Doyon O, White M, Rouleau JL, Brophy JM. Impact of care at a multidisciplinary congestive heart failure clinic: a randomized trial. CMAJ 2005;173(1):40-5.

9. Rich MW. Heart failure disease management programs: efficacy and limitations. Am 7 Med 2001;110:410-2.

10. Liu P, Arnold JM, Belenkie I, Demers C, Dorian P, Gianetti N, et al. The 2002/3 Canadian Cardiovascular Society consensus guideline update for the diagnosis and management of heart failure. Can F Cardiol 2003;19:347-56.

Correspondence to: Dr. Michael W. Rich, Cardiovascular Division, Washington University, 660 South Euclid Ave., Campus Box 8086, St. Louis MO 63110; fax: 314 454-5265;

mrich@im.wustl.edu

\section{Clinical trial registration}

CMAJ will consider clinical trials for publication only if they have been registered in a publicly accessible clinical trials registry before the enrolment of the first patient. This policy applies to trials that start recruiting on or after July 1, 2005. For trials that began enrolment before this date, registration is required by Sept. 13, 2005. The criteria for acceptable registration are described in CMAJ (2005;172 [13]:1700-2). 